\title{
Quinolone-Resistant Escherichia coli
}

Carmen Antonia Sanches Ito ${ }^{1,4}$, Ana Cristina Gales ${ }^{2}$, Maria Cristina B. Tognim ${ }^{2}$, Patrícia Munerato ${ }^{3}$ and Libera Maria Dalla Costa ${ }^{4}$ ${ }^{I}$ State University of Ponta Grossa (UEPG), Ponta Grossa, PR, Brazil $;{ }^{2}$ Alerta Laboratory, Federal University of São Paulo (UNIFESP), São Paulo, SP, Brazil; ${ }^{3}$ Retrovirology Laboratory - DIPA, Federal University of São Paulo (UNIFESP), São Paulo, SP, Brazil; ${ }^{4}$ Bacteriology Laboratory - SAC, Hospital de Clínicas, Federal University of Paraná (UFPR); Curitiba, PR, Brazil

\begin{abstract}
Quinolones (nalidixic acid - NAL, norfloxacin - NOR, ciprofloxacin - CIP and gatifloxacin - GAT) were tested against Escherichia coli isolated from urine (385 patient samples) by disk diffusion (DD) and agar dilution (AD) methods. Fifty-three samples $(\mathbf{1 3 . 8 \%})$ were classified as resistant to at least one of the quinolones tested. CIP and NOR susceptibilities were the same $(91.4 \%)$ and they were similar to GAT $(92.7 \%)$. Susceptibility to NAL, detected by the disk diffusion method, was used to predict susceptibility to NOR, CIP and GAT by the agar dilution method. The sensitivity and specificity of NAL were $100 \%$ and $95 \%$, respectively. Twelve samples were analyzed for mutations in the quinolone resistance-determining region (QRDR) of the gyrA and parC genes. Sequencing of these genes failed to find any mutations in the quinolone-sensitive isolates. However, three mutations were observed in the isolates resistant to all the quinolones tested - two in gyrA and one in parC. A single mutation in gyr A was found in the strains that were resistant to nalidixic acid but fluoroquinolone-sensitive. These findings support the suggestion that NAL could be used as a marker for susceptibility to fluoroquinolones in routine microbiology laboratories. The overall resistance rate to quinolones in the present study was $\mathbf{1 3 . 8 \%}$, which is higher than that observed in other studies carried out in developed countries. Our findings serve as a warning that resistance to this group of antimicrobial agents is increasing.

Key-Words: Urinary infection, quinolone resistance, Escherichia coli.
\end{abstract}

E. coli is the main etiologic agent of acute urinary tract infections [3,12], which are usually treated with quinolones and fluoroquinolones $[2,16]$. Nalidixic acid was the first clinically available quinolone. However, because of its pharmacokinetic properties and the emergence of resistant isolates, its clinical use has been abandoned [2], although it continues to be used in some Brazilian medical centers to treat urinary tract infections in the pediatric population.

The appearance of fluoroquinolone-resistant bacteria was observed immediately after the introduction of these antimicrobials into clinical practice to treat infections in hospitalized patients [16] and community-acquired infections caused by E. coli [1].

Resistance to fluoroquinolones occurs mainly as a result of mutations in bacterial gyr A and $\operatorname{par} \mathrm{C}$ genes that code for DNA gyrase and topoisomerase IV, respectively [14-16]. Single mutations in gyrA or par $\mathrm{C}$ can cause resistance to the older quinolones, such as nalidixic acid, as well as a reduction in the potency of fluoroquinolones, which is reflected in an increase in the minimal inhibitory concentration (MIC) $[6,8,17]$.

The aim of this study was to evaluate the antimicrobial activity of quinolones against $E$. coli isolated from urine as well as to establish if resistance to nalidixic acid could constitute a good marker of resistance to the newest fluoroquinolones. In addition, analyses to detect mutations Received on 5 September 2007; revised 28 January 2008.

Address for correspondence: Dr.Libera Maria Dalla Costa. Hospital de Clínicas - Universidade Federal do Paraná, Laboratório de Bacteriologia. Rua Padre Camargo, 280, $1^{\circ}$ Andar, Sala 203. Zip code: 80.060-240 Curitiba, Paraná, Brazil. Phone - 55-41-3360-7975 / Fax - 55-413360-1811. E-mail: 1mdc@ufpr.br.

The Brazilian Journal of Infectious Diseases

2008;12(1):5-9. (C) 2008 by The Brazilian Journal of Infectious Diseases and Contexto Publishing. All rights reserved. in the quinolone resistance-determining region (QRDR) of the gyrA and $\operatorname{par} \mathrm{C}$ genes were also carried out in selected isolates.

A total of 385 consecutive Escherichia coli isolates were obtained from the urine of patients. The patients selected exhibited urine quantitative counts $\left(\geq 10^{4} \mathrm{CFU} / \mathrm{mL}\right)$ and were examined by physicians at the Hospital de Clínicas, Universidade Federal do Paraná (HC-UFPR), between May and November - 2002. Only one bacterial isolate per patient was included in the study. These were identified using the WalkAway ${ }^{\circledR}$ automated system (Dade Behring ${ }^{\mathrm{TM}}$, Sacramento, USA).

The antibacterial activities of nalidixic acid (NAL), norfloxacin (NOR), ciprofloxacin (CIP), and gatifloxacin (GAT) were determined by agar dilution, according to the Clinical Laboratory Standards Institute (CLSI) guidelines [4]. Nalidixic acid susceptibility testing was also performed using the disk diffusion technique as described in the CLSI guidelines [5].

Molecular characterization of quinolone resistance was carried out by amplification of gyrA and $\operatorname{par} \mathrm{C}$ genes by PCR followed by sequencing of the respective amplicons [11,14] using the Big Dye Terminator Cycle Sequencing Ready Reaction kit (Applied Biosystems, Foster City, CA) and ABI BioPrism 3,100 DNA analyzer (Perkin-Elmer Applied Biosystems, Foster City, CA). Nucleotide sequences and their deduced protein product alignments were determined using the Lasergene software package (DNASTAR, Madison, WI). Most of the E. coli strains (91.2\%) were isolated from outpatients, and only $8.8 \%$ were isolated from hospitalized patients. The rate of susceptibility to CIP and NOR for the 385 E.coli isolates tested was the same $(91.4 \%)$ and was slightly lower than that for gatifloxacin (no longer on the market) $(92.7 \%)$ and higher than that for nalidixic acid (86.2\%).

Based on the agar dilution MIC results, the 385 E. coli isolates were divided into three groups: group 1, 332 (86.2\%) 
E. coli isolates susceptible to the four quinolones tested; group 2, 19 (4.9\%) E. coli isolates resistant to nalidixic acid but susceptible to the remaining quinolones; and group 3, 34 $(8.8 \%)$ E. coli isolates resistant to all four quinolones.

The ciprofloxacin MIC variations were evaluated among these three groups of strains according to patient gender (Table I). An interesting finding was that $E$. coli isolates belonging to group 3 were more frequently isolated from male than female patients $(\mathrm{p}<0.005)$. In group 1, CIP MICs varied from $\leq 0.03$ $\mu \mathrm{g} / \mathrm{mL}$ to $0.12 \mu \mathrm{g} / \mathrm{mL}$, as shown in Table 2 . In this group, 329 E. coli isolates exhibited CIP MICs $\leq 0.03 \mu \mathrm{g} / \mathrm{mL}$, two strains exhibited MICs of $0.06 \mu \mathrm{g} / \mathrm{mL}$, and one strain exhibited a MIC of $0.012 \mu \mathrm{g} / \mathrm{mL}$. As expected, the $34 \mathrm{E}$. coli isolates classified in group 3 had MICs higher than $2 \mu \mathrm{g} / \mathrm{mL}$. In group 2, there was a significant increase in the CIP MICs $(>0.06 \mu \mathrm{g} / \mathrm{mL})$ compared with group 1 ( $\mathrm{p}<0.005)$.

Twelve E. coli isolates (two randomly chosen from group 1 , five randomly chosen from group 2 and five randomly chosen from group 3) were submitted to genetic characterization of the quinolone resistance-determining region (QRDR) of the gyr A and parC genes. The mutations found in the QRDR of the gyrA and parC genes are shown in Table 2. The two $E$. coli isolates belonging to group $1 \mathrm{did}$ not show any mutations in the QRDR of the genes studied. However, the five isolates belonging to group 3 exhibited double mutations in the gyrA gene (codons 83 and 87) and a single mutation in the parC gene (codon 80). Of the five $E$. coli isolates belonging to group 2, three displayed a single mutation in the gyrA gene (codon 83). Curiously, one isolate from group 2 showed no mutation, while the remaining one showed a single mutation in the QRDR of the gyrA and parC genes.

A correlation was observed between NAL inhibition zones and minimal inhibitory concentrations for the fluoroquinolones (NOR, CIP, GAT), as illustrated in Figure 1. All the 333 E. coli isolates exhibiting NAL inhibition zones $\geq 19 \mathrm{~mm}$ were also susceptible to NOR, CIP and GAT. Among the $49 \mathrm{E}$. coli isolates resistant to NAL, 32, 33, and 31 isolates were categorized as resistant to NOR, CIP and GAT, respectively. The three E. coli isolates that exhibited intermediate resistance to NAL were susceptible to the fluoroquinolones tested.

For $99 \%$ of the E. coli isolates categorized as susceptible to NAL and CIP, the ciprofloxacin MICs were between 0.015 and $0.03 \mu \mathrm{g} / \mathrm{mL}$; however, in 13 of $15 \mathrm{E}$. coli isolates categorized as resistant to NAL and susceptible to CIP the corresponding figure was between 0.12 and $0.25 \mu \mathrm{g} / \mathrm{mL}$. The same pattern was observed for GAT and NOR. The MICs for $94 \%$ of the $E$. coli isolates susceptible to NAL and GAT were between 0.03 and $0.06 \mu \mathrm{g} / \mathrm{mL}$, while the MICs for isolates resistant to nalidixic acid but susceptible to gatifloxacin were above $0.25 \mu \mathrm{g} / \mathrm{mL}$. Thus, the $E$. coli isolates susceptible to NOR, CIP and GAT but with intermediate or full resistance to nalidixic acid showed an increase in fluoroquinolone MICs of $\geq 2$ dilutions compared with fully susceptible $E$. coli isolates. When susceptibility to nalidixic acid was assayed by disk diffusion to predict susceptibility to NOR, CIP and GAT, the sensitivity and specificity of nalidixic acid were $100 \%$ and $95 \%$, respectively. The negative predictive value (NPV) was $100 \%$ for the three quinolones studied, while the positive predictive value (PPV) was $65.4 \%$ for NOR and CIP and $63.5 \%$ for GAT.

The present study has shown higher rates of susceptibility to fluoroquinolones among urinary $E$. coli isolates collected from a Brazilian medical center than previously published studies that investigated Latin American isolates [12,18]. This difference may be because most of the $E$. coli in our study were isolated from outpatients rather than hospitalized patients.

Nalidixic acid inhibition zones correlated with the MICs of NOR, CIP and GAT. This finding shows that resistance to NAL can be used as a good indicator of fluoroquinolone resistance in E. coli. We observed that fluoroquinolone MICs for isolates that were resistant to NAL but susceptible to other fluoroquinolones were higher, indicating decreased susceptibility to these drugs. In addition, the level of resistance to quinolones depends on the number of accumulated mutations in the QRDR of the gyrA and parC genes [20]. Other resistance mechanisms, such as efflux pumps and alteration in outer membrane permeability, may also be involved.

A further interesting observation is that the number of nalidixic acid-resistant strains susceptible to fluoroquinolones was higher among female patients, while the number of $E$. coli isolates that were fully resistant to quinolones was higher in men, perhaps because of the association between urinary tract infections and prostatitis, where quinolones are widely used.

Bacteria with reduced susceptibility to quinolones commonly show mutations in the gyrA gene, which encodes the subunit gyrA of the DNA gyrase enzyme. These mutations occur in a region known as the "quinolone resistancedetermining region" (QRDR), which is located between nucleotides 201 and 320 and encodes amino acid residues 67 to 106 in E. coli gyrA. This is the main mechanism of quinolone-resistance in Enterobacteriaceae. Mutations in the gyrB gene have been reported, although they appear to play a minor role in conferring resistance to quinolones $[10,15,16]$. Mutations in the genes that encode topoisomerase IV, particularly the parC gene, correlate with reduced susceptibility to quinolones. Generally these mutations occur in amino acid positions 78, 80 and 84, with the substitution of glycine by aspartate, serine by arginine or isoleucine and glycine by aspartate, respectively. In the parE gene, mutations leading to quinolone resistance are not common, although the substitution of leucine by histidine has been detected at position $445[10,17,20]$.

In order to analyze the correlation between genetic characterization and resistance phenotype, ten quinoloneresistant and two quinolone-susceptible $E$. coli strains were submitted to amplification and sequencing of the gyrA and parC QRDR genes (Table 2). The isolates were divided into groups (1,2 and 3 ) according to the quinolone susceptibility 
Table 1. Distribution of ciprofloxacin minimal inhibiting concentrations in 385 E. coli isolates

\begin{tabular}{|c|c|c|c|c|c|c|c|c|c|c|c|c|c|c|c|c|}
\hline \multirow[t]{2}{*}{ Gender } & \multirow[t]{2}{*}{ Group } & \multicolumn{11}{|c|}{ Ciprofloxacin $(\mu \mathrm{g} / \mathrm{mL})$} & \multirow[b]{2}{*}{32} & \multirow[b]{2}{*}{64} & \multirow[b]{2}{*}{128} & \multirow[t]{2}{*}{$\mathbf{N}$} \\
\hline & & 0.015 & 0.03 & 0.06 & 0.125 & 0.25 & 0.5 & 1 & 2 & 4 & 8 & 16 & & & & \\
\hline & 1 & 218 & 72 & & & & & & & & & & & & & 290 \\
\hline \multirow[t]{2}{*}{ Female } & 2 & & 2 & 3 & 9 & 3 & & & & & & & & & & 17 \\
\hline & 3 & & & & & & & & & 1 & 3 & 2 & 7 & 3 & 4 & 20 \\
\hline \multirow[t]{3}{*}{ Male } & 1 & 30 & 9 & 2 & 1 & & & & & & & & & & & 42 \\
\hline & 2 & & & & 2 & & & & & & & & & & & 2 \\
\hline & 3 & & & & & & & & 1 & & & 4 & 6 & 1 & 2 & 14 \\
\hline
\end{tabular}

Group $1-E$. coli isolates susceptible to the four quinolones tested; group 2 - isolates resistant to nalidixic acid but susceptible to the other quinolones; and group 3 - isolates resistant to all four quinolones. $\mathrm{N}$ - number of isolates.

Table 2. Location and type of mutations in $\operatorname{gyr} A$ and $\operatorname{parC}$ genes found in selected $E$. coli isolates

\begin{tabular}{|c|c|c|c|c|c|}
\hline \multirow{4}{*}{ Strain } & \multirow{4}{*}{ Group } & \multicolumn{2}{|c|}{$\operatorname{MIC}(\mu \mathrm{g} / \mathrm{mL})$} & \multicolumn{2}{|c|}{ Mutation } \\
\hline & & \multirow{3}{*}{ NAL } & \multirow{3}{*}{ CIP } & gyrA & \multirow{2}{*}{$\frac{p a r C}{80}$} \\
\hline & & & & 87 & \\
\hline & & & & Ser Ala Val Tyr Asp & Asp Ser Ala \\
\hline 5384 & 1 & 1 & 0.03 & 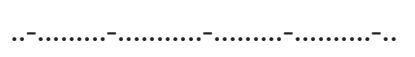 & ..-........................ \\
\hline 9850 & 1 & 1 & 0.015 & 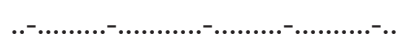 & ..-..........-.............. \\
\hline 837 & 2 & 64 & 0.03 & 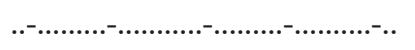 & ..-..........-............... \\
\hline 4633 & 2 & 64 & 0.25 & Leu......-....................-............. & ..-..........-.............. \\
\hline 9233 & 2 & 64 & 0.12 & Leu......-............-........-.............. & ..-.........-.............. \\
\hline 4557 & 2 & $>512$ & 0.12 & Leu......-...........-........-............. & ..-..........-.............. \\
\hline 8889 & 2 & $>512$ & 0.25 & Leu......-............-........-.............. & ..-........Arg.........-.. \\
\hline 6556 & 3 & $>512$ & 8 & Leu......-..........................Asn & ..-..........Ile..........- \\
\hline 345 & 3 & $>512$ & 64 & Leu......-..........................Asn & ..-........Ile..........- \\
\hline 6723 & 3 & $>512$ & 64 & Leu......-.........................Asn & ..-........Ile..........- \\
\hline 5850 & 3 & $>512$ & 4 & Leu......-.........................Asn & ..-........Ile...........- \\
\hline 6452 & 3 & $>512$ & 32 & Leu......-...........................Asn & ..-........Ile..........- \\
\hline
\end{tabular}

Group 1 - E. coli isolates susceptible to the four quinolones tested; group 2 - isolates resistant to nalidixic acid but susceptible to the other quinolones; and group 3 - isolates resistant to all four quinolones. NAL - nalidixic acid; CIP - ciprofloxacin.

phenotype; the MICs for ciprofloxacin of the isolates varied. Of the five strains belonging to Group 2, three $E$. coli isolates showed a single mutation in the gyrA gene at position 83 $(\mathrm{Ser} \rightarrow$ Leu) and one $E$. coli strain showed two mutations, one in $g y r \mathrm{~A}$ at position 83 ( $\mathrm{Ser} \rightarrow \mathrm{Leu}$ ) and another at position 80 of the $\operatorname{par} \mathrm{C}$ gene $(\mathrm{Ser} \rightarrow \mathrm{Arg})$. E. coli isolates with a single mutation in gyrA had increased fluoroquinolone MICs, but these were not high enough to indicate resistance according to the CLSI breakpoints. However, these isolates were fully resistant to nalidixic acid. These results are in agreement with those previously reported $[9,11]$, indicating that nalidixic acid could be a good marker for single mutations, even when testing is carried out by the disk diffusion method.
The $E$. coli strains belonging to group 3 had three mutations: two in the gyrA gene and one in the par $\mathrm{C}$ gene. This confirms that multiple mutations are necessary to express high levels of resistance to quinolones. The five strains resistant to CIP encoded three mutations, two in $g y r \mathrm{~A}$ at positions 83 (Ser $\rightarrow$ Leu) and 87 (Asp $\rightarrow$ Asn) and one in $\operatorname{par} C$ at position 80 ( $\mathrm{Ser} \rightarrow \mathrm{Ile}$ ). Only one $E$. coli isolate was resistant to nalidixic acid but susceptible to ciprofloxacin (MIC of $0.25 \mu \mathrm{g} / \mathrm{mL}$ ). It displayed two mutations, one in position 83 ( $\mathrm{Ser} \rightarrow$ Leu) of gyrA and the second at position 80 ( $\mathrm{Ser} \rightarrow \mathrm{Arg}$ ) of parC. This result, which was also observed by other authors, supports the supposition that it is necessary to accumulate mutations in the primary resistance gene to confer a high level of resistance to quinolones $[7,15,16]$. 
Figure 1. Correlation between inhibition zone of nalidixic acid (mm) and MICs $(\mu \mathrm{g} / \mathrm{mL})$ of norfloxacin, ciprofloxacin and gatifloxacin
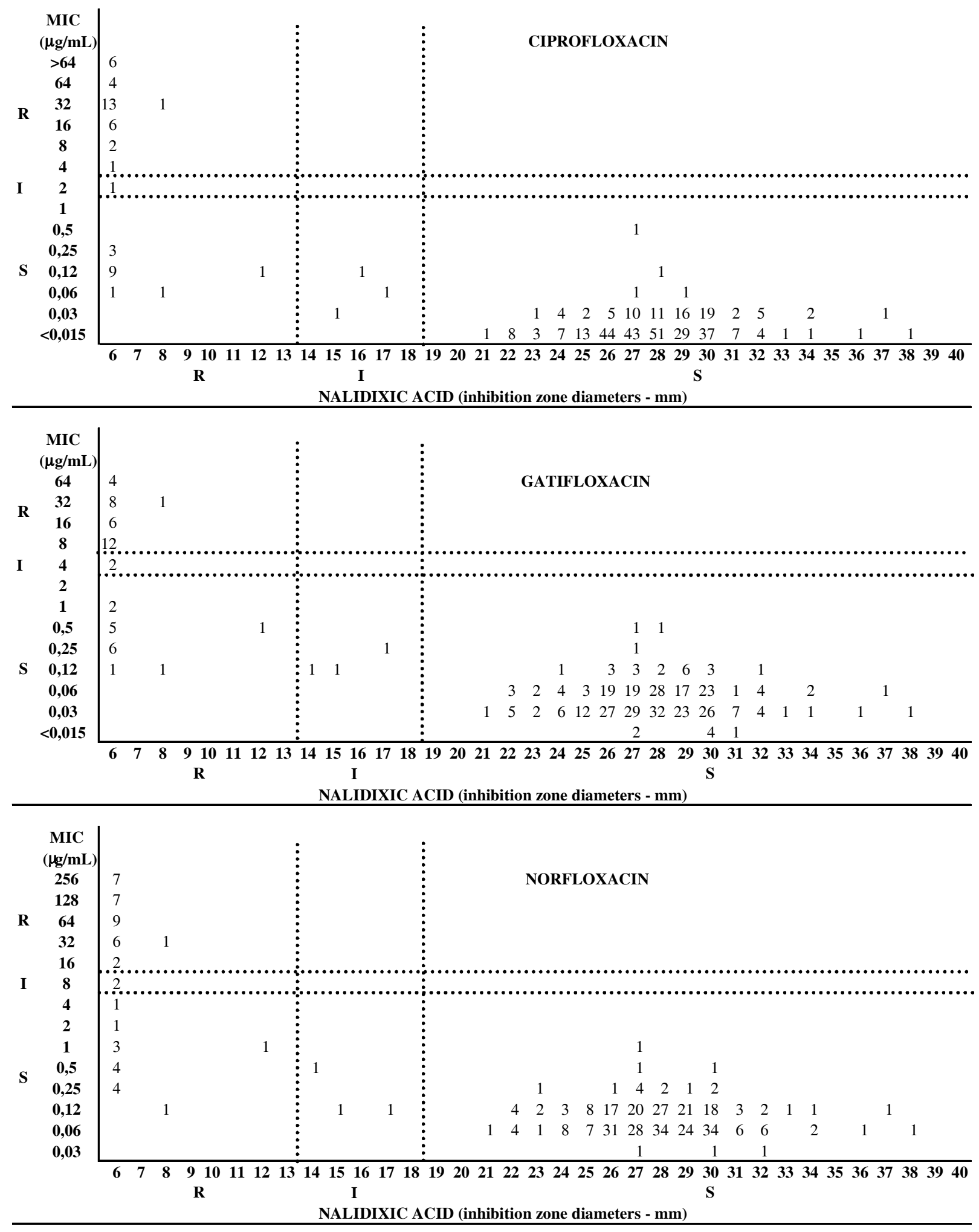
The overall resistance rate to quinolones in the present study was $13.8 \%$. Although this rate is lower than those reported by other Latin American studies, it is higher than those observed in other studies performed in developed countries $[12,19]$. Our findings serve as a warning that resistance to these groups of antimicrobial agents is increasing.

Testing for susceptibility to nalidixic acid was shown to be useful as a good predictive marker of susceptibility to fluoroquinolones in E. coli. The sensitivity of this screening method was as high as $100 \%$ with a specificity of $95 \%$. These results support the suggestion that nalidixic acid could be used as a marker for susceptibility to fluoroquinolones in routine microbiology laboratories.

\section{References}

1. Acar J.F., Goldstein F.W. Trends in: bacterial resistance to fluoroquinolones. Clin Infec Dis 1997;24:S67-73.

2. Andriole V.T. The quinolones: Academic Press, New York, 1998.

3. Berdichevski R.H., Zanardo J.C., Soilbelman M., Antonello I. Prevalência de uropatógenos e sua resistência aos antimicrobianos em uroculturas realizadas no Hospital São Lucas da PUCRS. Associação Médica do Rio Grande do Sul 1998;42:13742.

4. Clinical Laboratory Standards Institute CLSI. Methods for dilution antimicrobial susceptibility tests for bacteria that grow aerobically; Approved Standard - Sixth Edition, M7-A6. CLSI, Wayne, Pensylvania, USA, 2003.

5. Clinical Laboratory Standards Institute CLSI. Performance Standards for Antimicrobial Disk Susceptibility Tests; Approved Standard - Eighth Edition. M2-A8. CLSI, Wayne, Pensylvania, USA, 2003.

6. Drlica K., Zhao X. DNA gyrase, topoisomerase IV and the 4 quinolones. Microbiol Mol Biol Rev 1997;61:377-92.

7. Drlica K. Mechanism of fluoroquinolone action. Curr Opin Microbiol 1999;2:504-8.

8. Drlica K. A strategy for fighting antibiotic resistance. ASM News 2001; 67: 27-33.
9. Ena J., López-Perezagua M.M., Martínez-Peinado C., Cia-Barrio M.A., Ruíz-López I. Emergence of ciprofloxacin resistance in Escherichia coli isolates after widespread use of fluoroquinolones. Diagn Microbiol Infect Dis 1998;30:103-7.

10. Everett M., Jin Y.F., Ricci V., Piddock L.J.V. Contributions of individual mechanisms to fluoroquinolone resistance in 36 Escherichia coli strains isolated from humans and animals. Antimicrob Agents Chemother 1996;40:2380-6.

11. Gales A.C., Gordon K.A., Wilke W.W., Pfaller M.A., Jones R.N. Occurrence of single-point IA mutations among ciprofloxacinsusceptible Escherichia coli. Diagn Microbiol Infect Dis 2000a:36:61-4.

12. Gales A.C., Jones R.N., Gordon K.A., et al. Activity and spectrum of 22 antimicrobial agents tested against urinary tract infection pathogens in hospitalized patients in Latin America: report from the second year of the SENTRY Antimicrobial Surveillance Program (1998). J Antimicrob Chemother 2000b;45:1295-303.

13. Georgiou M., Muñoz R., Román F., et al. Ciprofloxacin-resistant Haemophilus influenzae strains possess mutations in analogous positions of GyrA and ParC. Antimicrob Agents Chemother 1996; $40: 1741-4$.

14. Heisig P. Genetic evidence for a role of $\operatorname{Par} \mathrm{C}$ mutations in development of high-level fluoroquinolone resistance in Escherichia coli. Antimicrob Agents Chemother 1996;40:879-85.

15. Hooper D.C. Bacterial topoisomerases, anti-topoisomerases and anti-topoisomerase resistance. Clin Infect Dis 1998;27:54-63.

16. Hooper D.C. Emerging mechanisms of fluoroquinolone resistance. Emerg Infect Dis 2001;7:337-41.

17. Kern W.V., Steib-Bauert M., de With K., et al. Fluoroquinolone consumption and resistance in hematology-oncology patients: ecological analysis in two university hospitals 1999-2002. J Antimicrob Chemother 2005;55:57-60.

18. Lopes A.A., Salgado K., Martinelli R., Rocha H. Increase in the frequency of norfloxacin and ciprofloxacin resistance of bacteria isolated from urine culture. Revista da Associação Médica do Brasil 1998;44:196-200.

19. Sanders C.C. Mechanisms responsible for cross-resistance and dichotomous resistance among the quinolones. Clin Infect Dis 2001;32:S1-S8.

20. Vila J., Ruiz J., Marco F., et al. Association between double mutation in GyrA gene of ciprofloxacin-resistant clinical isolates of $E$. coli and MICs. Antimicrob Agents Chemother 1994;38:2477-79. 